PROCEEDINGS OF THE

AMERICAN MATHEMATICAL SOCIETY

Volume 140, Number 1, January 2012, Pages 367-376

S 0002-9939(2011)10917-1

Article electronically published on May 26, 2011

\title{
ON THE CROSSING NUMBERS OF A VIRTUAL KNOT
}

\author{
SHIN SATOH AND YUMI TOMIYAMA
}

(Communicated by Daniel Ruberman)

\begin{abstract}
We give lower bounds of the real crossing number of a virtual knot in terms of the Jones polynomial and the Miyazawa polynomial. As an application, we prove the existence of a virtual knot such that the real and virtual crossing numbers are equal to $m$ and $n$ for any positive integers $m<n$.
\end{abstract}

\section{INTRODUCTION}

Virtual knot theory [8] studies the embeddings of circles in thickened surfaces of arbitrary genus up to the addition and removal of empty handles from the surface. Virtual links are represented by diagrams in the plane by introducing virtual crossings as well as real crossings.

For a virtual link $L$, there are two crossing numbers - the minimal number $\mathrm{r}(L)$ of real crossings and the minimal number $\mathrm{v}(L)$ of virtual crossings (cf. 3] ). For a classical link $L$, there is also the ordinary crossing number $\mathrm{c}(L)$ - the minimal number of real crossings for all "classical" diagrams of $L$. We remark that $\mathrm{c}(L) \geq$ $\mathrm{r}(L)$ holds by definition.

To find several properties on these crossing numbers, we use the Jones polynomials and the Miyazawa polynomials. The Jones polynomial $V_{L}(t)$ was defined for classical links originally and was extended to virtual links by the state sum model 8]. The Miyazawa polynomial $R_{L}(A, \boldsymbol{x})$ is a generalization of the Jones polynomial defined by the enhanced states [13] and is equivalent to the arrow polynomial due to Dye and Kauffman [2].

In classical category, it is well-known that the crossing number is greater than or equal to the span of the Jones polynomial

$$
\mathrm{c}(L) \geq \operatorname{span} V_{L}(t)
$$

for any non-splittable classical link $L$ [7, 15, 16]. By a similar argument, we see that the real crossing number satisfies the same property

$$
\mathrm{r}(L) \geq \operatorname{span} V_{L}(t)
$$

in virtual category, and $\mathrm{r}(L)=\mathrm{c}(L)$ holds for any classical alternating links (Section 21). It is unknown whether the equality also holds for any non-alternating links.

Received by the editors March 25, 2010 and, in revised form, November 14, 2010.

2010 Mathematics Subject Classification. Primary 57M25; Secondary 57M27.

Key words and phrases. Virtual knot, crossing number, Jones polynomial, Miyazawa polynomial, arrow polynomial.

(C)2011 American Mathematical Society Reverts to public domain 28 years from publication 
On the other hand, there are lower bounds of the real and virtual crossing numbers by the weighted degree of the Miyazawa polynomial

$$
\mathrm{r}(L), \mathrm{v}(L) \geq \operatorname{wdeg} R_{L}(A, \boldsymbol{x})
$$

for any virtual link $L$ [2, 14. In Section 3, we improve the lower bound of the real crossing number as

$$
\mathrm{r}(K) \geq \operatorname{wdeg} R_{K}(A, \boldsymbol{x})+1
$$

for any virtual knot $K$. We can find other studies on the crossing numbers in [1, 11, 12, for example.

Very little is known about the relationship between the real and virtual crossing numbers. It is conjectured that $\mathrm{v}(L) \leq \mathrm{r}(L)$ for any virtual link $L$ and that $\mathrm{v}(K)<$ $\mathrm{r}(K)$ for any virtual knot $K$. We remark that this inequality holds in the case of $\mathrm{r}(K) \leq 4$ by checking the virtual knot table due to Green 44. In Section 4, we prove that for any positive integers $m<n$ there is a virtual knot $K$ satisfying $\mathrm{v}(K)=m$ and $\mathrm{r}(K)=n$. Ishii [5] proves that the 2-component virtual link $L$ as shown in Figure 1 satisfies $\mathrm{v}(L)=\mathrm{r}(L)=n$.

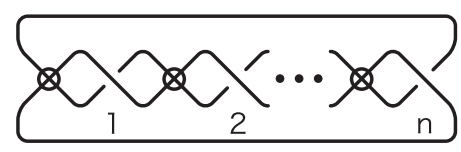

\section{Figure 1}

In the last section, we find several properties of the coefficients of the Miyazawa polynomials.

\section{The Jones polynomial $V_{L}(t)$}

We recall the definition of the Jones polynomial of a virtual link $L$ (cf. [8]). Let $D$ be a diagram of $L$. The writhe $w(D)$ is the number of positive crossings of $D$ minus that of negative crossings. A state $S$ is a diagram obtained from $D$ by replacing every real crossing $\mathcal{X}$ with the $A$-splicing $\asymp$ or the $B$-splicing ) (. The result is a union of immersed loops with virtual crossings. Let $|S|$ denote the number of loops of $S$, and let $a(S)$ and $b(S)$ be the numbers of $A$-and $B$-splicings for $S$, respectively.

We define the bracket polynomial $\langle S \mid D\rangle \in \mathbb{Z}\left[A, A^{-1}\right]$ by

$$
\langle S \mid D\rangle=A^{a(S)-b(S)}\left(-A^{2}-A^{-2}\right)^{|S|-1} .
$$

The polynomial $f_{D}(A)$ has the form of a state sum such that

$$
f_{D}(A)=\left(-A^{3}\right)^{-w(D)} \sum_{S}\langle S \mid D\rangle .
$$

Then $V_{D}(t)=f_{D}\left(t^{-\frac{1}{4}}\right)$ is an invariant of $L$. We call it the Jones polynomial and denote it by $V_{L}(t)$. The span of $V_{L}(t)$ is the difference between the maximal and minimal degrees with respect to the variable $t$ and is denoted by $\operatorname{span} V_{L}(t)$.

Let $r(D)$ and $v(D)$ denote the numbers of real and virtual crossings of $D$, respectively. A virtual link diagram $D$ is classical if $v(D)=0$ and is alternating if it is classical and alternating in the ordinary sense. Furthermore, a virtual link $L$ 
is classical or alternating if it is represented by a classical or alternating diagram, respectively.

The real and virtual crossing numbers of a virtual link $L$ are the minimal numbers of $r(D)$ and $v(D)$ for all diagrams $D$ of $L$ and are denoted by $\operatorname{r}(L)$ and $\mathrm{v}(L)$, respectively. For a classical link $L$, the crossing number $\mathrm{c}(L)$ in the ordinary sense is the minimal number of $r(D)$ for all "classical" diagrams $D$ of $L$.

We say that $D$ is a split diagram if there are two disjoint disks $\Delta_{i}$ in the plane with $D \cap \partial \Delta_{i}=\emptyset$ and $L \cap \Delta_{i} \neq \emptyset(i=1,2)$. A virtual link $L$ is non-splittable if it has no split diagram.

Theorem 2.1. (i) If a virtual link $L$ is non-splittable, then it holds that

$$
\mathrm{r}(L) \geq \operatorname{span} V_{L}(t) .
$$

(ii) Let $L$ be a non-splittable alternating link and $D$ be a reduced alternating diagram of $L$. Then it holds that

$$
\mathrm{r}(L)=r(D)=\operatorname{span} V_{L}(t) .
$$

Therefore, we have $\mathrm{r}(L)=\mathrm{c}(L)$ for any (possibly splittable) alternating link $L$.

(iii) Let $D$ be a reduced alternating diagram, $D^{\prime}$ a diagram obtained from $D$ by replacing a single real crossing with a virtual crossing, and $L^{\prime}$ the virtual knot represented by $D^{\prime}$. Then it holds that

$$
\mathrm{r}\left(L^{\prime}\right)=r\left(D^{\prime}\right)=\operatorname{span} V_{L^{\prime}}(t)+\frac{1}{2} .
$$

Therefore, $L^{\prime}$ is a non-classical virtual link.

Proof. Most part of the theorem is proved the same as the classical case (cf. 7]). The second equality in (iii) has been proved in [6]. We just notice that

$$
\left|S_{A}^{\prime}\right|+\left|S_{B}^{\prime}\right|=r\left(D^{\prime}\right)+1
$$

where $S_{A}^{\prime}$ (or $S_{B}^{\prime}$ ) is the state of $D^{\prime}$ obtained by $A$ - (or $B$-)splicing at every real crossing.

In [1], Manturov proves the inequality $r(D)+\frac{1}{2} \chi(D)-1 \geq \operatorname{span} V_{L}(t)$, where $\chi(L)$ is the Euler characteristic of the atom (the Turaev surface [17]) associated with $D$. Since $\chi(D) \leq 2$, we have $r(D) \geq \operatorname{span} V_{L}(t)$. In particular, it is easy to see that $\chi(D)=2$ and $\chi\left(D^{\prime}\right)=1$ in (ii) and (iii), respectively.

We have the following property of the Jones polynomial by skein relations immediately.

Lemma 2.2 (cf. 9]). The local deformations for virtual link diagrams as shown in Figure 2 do not change the Jones polynomial.
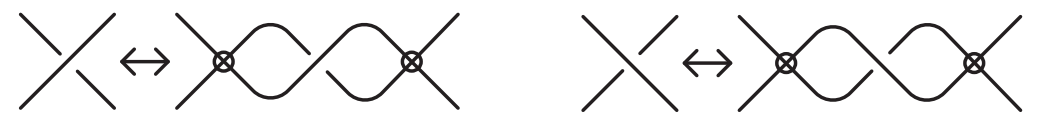

FiguRE 2

The local deformation in Lemma 2.2 is called virtualization. 
Example 2.3. Let $K_{i}$ be the virtual knot represented by the diagram $D_{i}(i=$ $1,2,3)$ as shown in Figure 3. It holds that $V_{K_{1}}(t)=V_{K_{2}}(t)$ by Lemma 2.2. Since $D_{2}$ is obtained from the reduced alternating diagram $D_{3}$ by replacing a single real crossing with a virtual crossing, it holds that $\operatorname{span} V_{K_{2}}(t)=r\left(D_{2}\right)-\frac{1}{2}=\frac{7}{2}$ by Theorem 2.1(iii). Therefore, we have $\mathrm{r}\left(K_{1}\right)=4$ by Theorem 2.1(i). The virtual knot $K_{1}$ is found in Green's table [4] as 4.80 .

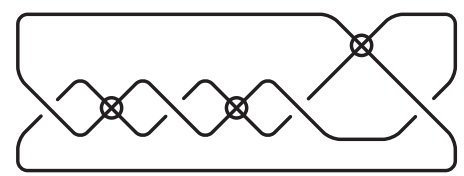

$\mathrm{D}_{1}$

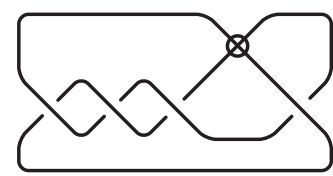

$\mathrm{D}_{2}$

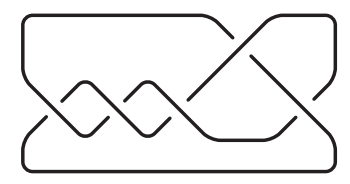

D3

Figure 3

\section{The Miyazawa polynomial $R_{L}(A, \boldsymbol{x})$}

We introduce the Miyazawa polynomial [13] by using enhanced states due to Ishii [5] (cf. 6]). In an enhanced state $S$ of $D$, every real crossing $\$ is spliced coherently $\asymp$ or non-coherently $)-($ with respect to the orientation of $D$, where we put a pair of short poles for every non-coherent splicing. We remark that each loop of $S$ has an even number of poles.

For a loop $C$ of $S$, we define an index $\lambda(C)$ as follows:

- We may slide a pole along $C$ through a virtual crossing $\mathbf{\perp} \boldsymbol{\phi} \leftrightarrow \boldsymbol{\phi} \mathbf{\phi}$.

- If an adjacent pair of poles is on the same side of $C$, then we remove the pair $\mu \rightarrow$ —.

- The result is a collection of alternating poles number of them is denoted by $\lambda(C)$.

For a non-negative integer $i$, we denote by $\lambda_{i}(S)$ the number of loops of $S$ of index $i$. The equality $\sum_{i>0} \lambda_{i}(S)=|S|$ holds by definition.

Let $a(S)$ and $b(S)$ denote the numbers of $A$ - and $B$-splicings for $S$, respectively. We define the enhanced bracket polynomial $\langle S \mid D\rangle^{\prime} \in \mathbb{Z}\left[A, A^{-1}, x_{1}, x_{2}, \ldots\right]$ by

$$
\langle S \mid D\rangle^{\prime}=A^{a(S)-b(S)}\left(-A^{2}-A^{-2}\right)^{\lambda_{0}(S)} x_{1}^{\lambda_{1}(S)} x_{2}^{\lambda_{2}(S)} \ldots
$$

The polynomial $R_{D}(A, \boldsymbol{x})$ has the form of a state sum such that

$$
R_{D}(A, \boldsymbol{x})=\left(-A^{3}\right)^{-w(D)} \sum_{S}\langle S \mid D\rangle^{\prime}
$$

where $\boldsymbol{x}=\left(x_{1}, x_{2}, \ldots\right)$. The polynomial $R_{D}(A, \boldsymbol{x})$ is an invariant of $L$. We call it the Miyazawa polynomial and denote it by $R_{L}(A, \boldsymbol{x})$. The Miyazawa polynomial is a generalization of the Jones polynomial; in fact, by putting $x_{i}=-A^{2}-A^{-2}$ for any $i \geq 1$ in $R_{L}(A, \boldsymbol{x})$, we obtain $\left(-A^{2}-A^{-2}\right) f_{L}(A)$.

We denote by

$$
F_{\lambda_{1} \lambda_{2} \ldots \lambda_{n}}(A) \in \mathbb{Z}\left[A, A^{-1}\right]
$$


the coefficient of the term of $R_{L}(A, \boldsymbol{x})$ with respect to $\boldsymbol{x}$ containing $x_{1}^{\lambda_{1}} x_{2}^{\lambda_{2}} \ldots x_{n}^{\lambda_{n}}$ $\left(\lambda_{n} \neq 0\right)$ and by $F_{0}(A)$ the constant term of $R_{L}(A, \boldsymbol{x})$. We often use the expression

$$
R_{L}(A, \boldsymbol{x})=\sum_{\lambda} F_{\lambda}(A) \boldsymbol{x}^{\lambda}
$$

where $\boldsymbol{x}^{\lambda}=x_{1}^{\lambda_{1}} x_{2}^{\lambda_{2}} \ldots x_{n}^{\lambda_{n}}$ for $\lambda=\left(\lambda_{1}, \lambda_{2}, \ldots, \lambda_{n}\right)$.

The weighted degree of a term $F_{\lambda}(A) \boldsymbol{x}^{\lambda}$ is equal to $\sum_{i>1} i \cdot \lambda_{i}$, and the weighted degree of the polynomial $R_{L}(A, \boldsymbol{x})$ is the maximal degree for all the terms (cf. [13]). We denote it by wdeg $R_{L}(A, \boldsymbol{x})$.

Theorem 3.1 (cf. [2, 13]). For a virtual link L, it holds that

(i) $\mathrm{v}(L) \geq \operatorname{wdeg} R_{L}(A, \boldsymbol{x})$ and

(ii) $\mathrm{r}(L) \geq \operatorname{wdeg} R_{L}(A, \boldsymbol{x})$.

In the case of a virtual knot, we have a better lower bound of the real crossing number as follows.

Proposition 3.2. For a non-trivial virtual knot $K$, it holds that

$$
\mathrm{r}(K) \geq \operatorname{wdeg} R_{K}(A, \boldsymbol{x})+1 .
$$

Proof. Put $n=\mathrm{r}(K)$. Assume that $\operatorname{wdeg} R_{K}(A, \boldsymbol{x})=n$. Let $D$ be a diagram of $K$ with $r(D)=n$. By assumption, there is a state $S$ of $D$ such that $\sum_{i \geq 1} i \cdot \lambda_{i}(S)=n$. It follows by $\sum_{C} \lambda(C)=n$ that every real crossing is spliced non-coherently in $S$ and that the poles appear alternately along each loop of $S$.

We label the real crossings with $1,2, \ldots, n$ and the endpoints of the arcs around the $k$ th crossing with $a_{k}, b_{k}, c_{k}$, and $d_{k}$ as shown in Figure 4. Then $a_{k}$ must connect with some $d_{l}$ in $S$ and $b_{k}$ must connect with some $c_{m}$. Therefore, the segments $a_{k} d_{k}$ and $c_{k} b_{k}$ in $D$ belong to different components of $K$. This contradicts the fact that $K$ is a knot, and we have $\operatorname{wdeg} R_{K}(A, \boldsymbol{x})>n$ by Theorem 3.1 .
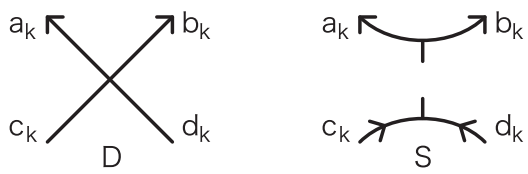

Figure 4

Example 3.3. Let $K_{1}$ be the virtual knot in Example 2.3. The Miyazawa polynomial of $K_{1}$ is given by

$$
R_{K_{1}}(A, \boldsymbol{x})=F_{0}(A)+F_{1}(A) x_{1}+F_{001}(A) x_{3}+F_{11}(A) x_{1} x_{2},
$$

where

$F_{0}(A)=-A^{14}-A^{2}, F_{1}(A)=A^{14}-A^{10}+A^{6}, F_{001}(A)=A^{14}$, and $F_{11}(A)=A^{16}$.

Since $\operatorname{wdeg} R_{K_{1}}(A)=3$, we have $\mathrm{v}\left(K_{1}\right)=3$ by Theorem 3.1(i) and $\mathrm{r}\left(K_{1}\right)=4$ by Proposition 3.2 


\section{4. $\mathrm{v}(K)$ vS. $\mathrm{r}(K)$}

The aim of this section is to prove the following theorem.

Theorem 4.1. For any positive integers $m$ and $n$ with $m<n$, there is a virtual knot $K$ such that

(i) $\mathrm{v}(K)=m$ and

(ii) $\mathrm{r}(K)=n$.

We will prove this theorem by using three lemmas. Let $=\mathrm{c} \in$ denote the 2string tangle $\lambda \mathbf{C} \cdot \boldsymbol{X}$ with $c$ crossings. We have the following lemma by skein relations immediately, where $\langle D\rangle^{\prime}=\sum_{S}\langle S \mid D\rangle^{\prime}$ for a diagram $D$.

Lemma 4.2. For a positive integer $c$, it holds that

$$
\langle\text { 二c }=\rangle^{\prime}=A^{c}\langle\asymp\rangle^{\prime}+A^{c-2} \frac{1-\left(-A^{-4}\right)^{c}}{1+A^{-4}}\langle)(\rangle^{\prime},
$$

where $\asymp$ or $)$ ( on the right-hand side may have the pair of poles according to the orientation of the original tangle.

Lemma 4.3. Let $a$ and $b$ be integers with $a \geq 0, b \geq 2$, and $b$ even. Let $K_{1}(a, b)$ be the virtual knot represented by the diagram $D$ as shown in Figure 5. Then we have

(i) $\mathrm{v}\left(K_{1}(a, b)\right)=a+1$ and

(ii) $\mathrm{r}\left(K_{1}(a, b)\right)=a+b$.

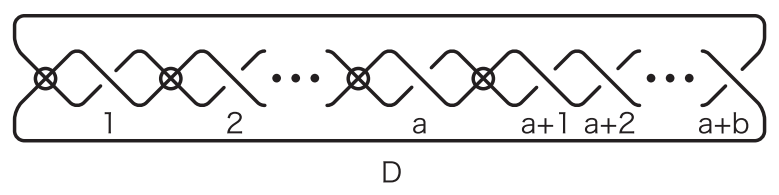

Figure 5

Proof. (i) By Theorem 3.1 it is sufficient to prove that wdeg $\langle D\rangle^{\prime}=a+1$. Let $E$ and $F$ be the diagrams as shown in Figure 6. By Lemma 4.2, it holds that

$$
\langle D\rangle^{\prime}=A^{b}\langle E\rangle^{\prime}+A^{b-2} \frac{1-A^{-4 b}}{1+A^{-4}}\langle F\rangle^{\prime} .
$$

Since $r(E)=a$, we have wdeg $\langle E\rangle^{\prime} \leq a-1$ by Proposition 3.2 . On the other hand, $\langle F\rangle^{\prime}$ has the unique term of weighted degree $a+1$ obtained by splicing every real crossing non-coherently; in fact, the term is

$$
F_{a+1}(A) x_{1}^{a+1}=A^{-a+b-2} \frac{1-A^{-4 b}}{1+A^{-4}} x_{1}^{a+1} .
$$

Therefore, we have $\mathrm{wdeg}\langle D\rangle=\mathrm{wdeg}\langle F\rangle=a+1$.

(ii) By Theorem 2.1(i), it is sufficient to prove that $\operatorname{span} V_{D}(t)=a+b$ or $a+b-\frac{1}{2}$. We perform the local deformations in Lemma 2.2 for $D$ repeatedly so that we obtain a diagram $G$ as shown on the left (for $a$ odd) or right (for $a$ even) of Figure 7. Therefore, we have

$$
\operatorname{span} V_{D}(t)=\operatorname{span} V_{G}(t)= \begin{cases}a+b & (a: \text { odd }) \\ a+b-\frac{1}{2} & (a: \text { even })\end{cases}
$$



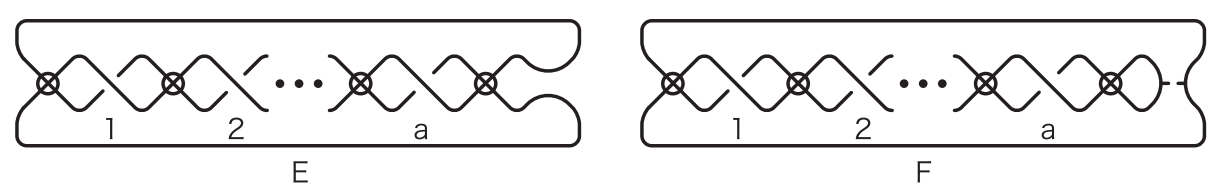

FiguRE 6

by Theorem 2.1(ii) and (iii).

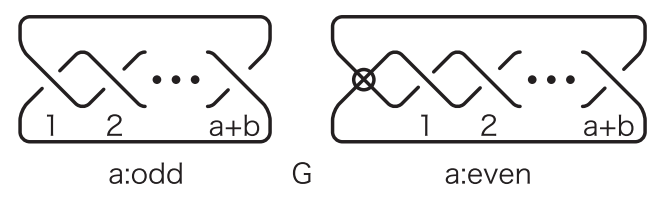

FiguRE 7

The following lemma is proved similarly to Lemma 4.3, and we omit the proof.

Lemma 4.4. Let $a$ and $b$ be integers with $a \geq 1, b \geq 2$, and $b$ even. Let $K_{2}(a, b)$ be the virtual knot represented by the diagram $D$ as shown in Figure 8 . Then we have

(i) $\mathrm{v}\left(K_{2}(a, b)\right)=a$ and

(ii) $\mathrm{r}\left(K_{2}(a, b)\right)=a+b$.

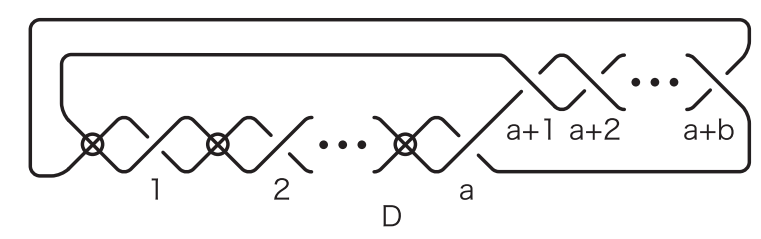

FigURE 8

Proof of Theorem 4.1. If $m$ and $n$ have opposite parities, then we may take $K=$ $K_{1}(m-1, n-m+1)$. If $m$ and $n$ have the same parity, then we may take $K=$ $K_{2}(m, n-m)$. Then $K$ satisfies the conditions in the theorem by Lemmas 4.2 and 4.3 .

\section{The coefficient polynomial $F_{\lambda}(A)$}

We conclude this paper with several properties of the coefficient $F_{\lambda}(A)$ of the Miyazawa polynomial $R_{L}(A, \boldsymbol{x})=\sum_{\lambda} F_{\lambda}(A) \boldsymbol{x}^{\lambda}$ with $\lambda=\left(\lambda_{1}, \lambda_{2}, \ldots\right)$.

Proposition 5.1. Let $L$ be a virtual link with $\mu(L)$ components:

(i) $F_{\lambda}(A) \in \mathbb{Z}\left[A^{4}, A^{-4}\right] \cdot A^{2 \mu(L)}$ if and only if $\sum_{i \geq 1} \lambda_{i} \equiv 0(\bmod 2)$.

(ii) $F_{\lambda}(A) \in \mathbb{Z}\left[A^{4}, A^{-4}\right] \cdot A^{2 \mu(L)+2}$ if and only if $\sum_{i \geq 1} \lambda_{i} \equiv 1(\bmod 2)$. 
We will prove this proposition by using two lemmas. For a state $S$, we put

$$
d(S)=-3 w(D)+a(S)-b(S)+2 \lambda_{0}(S) .
$$

Let $\nu(S)$ denote the number of non-coherent splicings for $S$ and $S_{0}$ denote the unique state of $D$ with $\nu\left(S_{0}\right)=0$. We remark that $\lambda_{0}\left(S_{0}\right)=\left|S_{0}\right|$ and $\lambda_{i}\left(S_{0}\right)=0$ for any $i \geq 1$.

Lemma 5.2. $d\left(S_{0}\right) \equiv 2 \mu(L)(\bmod 4)$.

Proof. If a virtual link $L^{\prime}$ is obtained from $L$ by splicing a real crossing coherently, then it holds that $\mu\left(L^{\prime}\right)=\mu(L) \pm 1$. Therefore, we have the equality

$$
\left|S_{0}\right| \equiv r(D)+\mu(L)(\bmod 2)
$$

by induction on the number $r(D)$ of real crossings. Since $a\left(S_{0}\right)-b\left(S_{0}\right)=w(D)$ and $w(D) \equiv r(D)(\bmod 2)$, it holds that

$$
d\left(S_{0}\right)=-2 w(D)+2\left|S_{0}\right| \equiv 2 \mu(L)(\bmod 4) .
$$

Let $S$ and $S^{\prime}$ be two states of $D$ such that $S^{\prime}$ is obtained from $S$ by replacing a coherent splicing with a non-coherent one at a single real crossing. We remark that $\nu\left(S^{\prime}\right)=\nu(S)+1$.

Lemma 5.3. (i) $d\left(S^{\prime}\right)-d(S) \equiv 2\left[\lambda_{0}\left(S^{\prime}\right)-\lambda_{0}(S)\right]+2(\bmod 4)$.

(ii) $\sum_{i \geq 0} \lambda_{2 i}\left(S^{\prime}\right)-\sum_{i \geq 0} \lambda_{2 i}(S) \equiv 1(\bmod 2)$.

Proof. (i) The congruence follows by $a\left(S^{\prime}\right)-a(S)= \pm 1$ and $b\left(S^{\prime}\right)-b(S)=\mp 1$.

(ii) Since $|S|-1 \leq\left|S^{\prime}\right| \leq|S|+1$, we divide the proof into three cases. We consider the case $\left|S^{\prime}\right|=|S|-1$ only, and the other cases are similarly checked.

Let $C_{1}, C_{2}$, and $C^{\prime}$ be the loops such that $C_{1} \cup C_{2}$ in $S$ is replaced with $C^{\prime}$ in $S^{\prime}$. Then there are two cases to consider as shown in Figure 9. Since $\sum_{i \geq 0} \lambda_{2 i}(S)$ is the number of loops with even index, we have the conclusion.

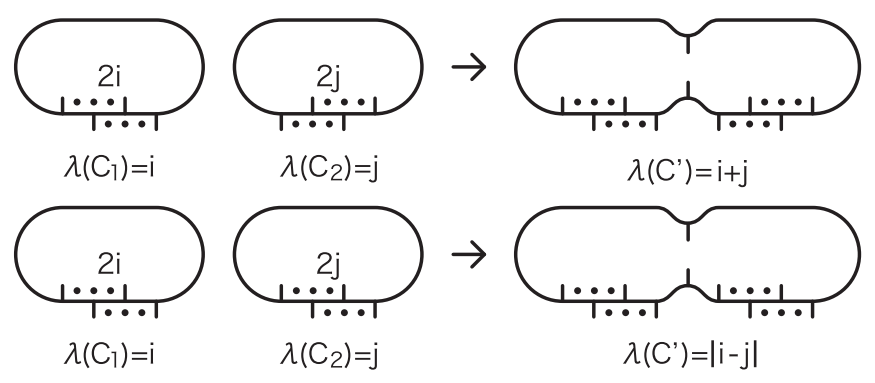

FIGURE 9

Proof of Proposition 5.1. Recall that the Miyazawa polynomial $R_{L}(A, \boldsymbol{x})$ is the sum of

$$
\left(-A^{3}\right)^{-w(D)}\langle S \mid D\rangle^{\prime}=\left(-A^{3}\right)^{-w(D)}\left(-A^{2}-A^{-2}\right)^{\lambda_{0}(S)}
$$

for all states $S$ of $D$. The polynomial $\left(-A^{3}\right)^{-w(D)}\langle S \mid D\rangle^{\prime}$ has the maximal degree $d(S)$ and belongs to $\mathbb{Z}\left[A^{4}, A^{-4}\right] \cdot A^{d(S)}$. Therefore, it is sufficient to prove that

$$
d(S) \equiv 2 \sum_{i \geq 1} \lambda_{2 i}(S)+2 \mu(L)(\bmod 4) .
$$


We prove it by induction on $\nu(S)$. The case $\nu(S)=0$ follows by Lemma 5.2 . For any states $S$ and $S^{\prime}$ in Lemma 5.3. if $S$ satisfies the congruence, then so does $S^{\prime}$. In fact, it follows by Lemma 5.3 that

$$
\begin{aligned}
d\left(S^{\prime}\right) & \equiv d(S)+2\left[\lambda_{0}\left(S^{\prime}\right)-\lambda_{0}(S)\right]+2 \\
& \equiv\left[2 \sum_{i \geq 1} \lambda_{2 i}(S)+2 \mu(L)\right]+2 \lambda_{0}(S)+2 \lambda_{0}\left(S^{\prime}\right)+2 \\
& \equiv 2\left[\sum_{i \geq 0} \lambda_{2 i}\left(S^{\prime}\right)+1\right]+2 \mu(L)+2 \lambda_{0}\left(S^{\prime}\right)+2 \\
& \equiv 2 \sum_{i \geq 1} \lambda_{2 i}\left(S^{\prime}\right)+2 \mu(L)(\bmod 4) .
\end{aligned}
$$

Dye and Kauffman 2] define the arrow polynomial by

$$
\langle L\rangle_{N A}=\left(-A^{3}\right)^{-w(D)} \sum_{S} A^{a(S)-b(S)}\left(-A^{2}-A^{-2}\right)^{|S|-1} K_{1}^{\lambda_{1}(S)} K_{2}^{\lambda_{2}(S)} \cdots
$$

in $\mathbb{Z}\left[A, A^{-1}, K_{1}, K_{2}, \ldots\right]$. It is easy to see that the arrow polynomial is equivalent to the Miyazawa polynomial. In fact, $\langle L\rangle_{N A}$ is obtained from $R_{L}(A, \boldsymbol{x})$ by replacing each $x_{i}$ with $\left(-A^{2}-A^{-2}\right) K_{i}$ and dividing the result by $-A^{2}-A^{-2}$. We use the expression $\langle L\rangle_{N A}=\sum_{\lambda} G_{\lambda}(A) K^{\lambda}$ with $K^{\lambda}=K_{1}^{\lambda_{1}} K_{2}^{\lambda_{2}} \ldots$ for $\lambda=\left(\lambda_{1}, \lambda_{2}, \ldots\right)$ as well as $R_{L}(A, \boldsymbol{x})=\sum_{\lambda} F_{\lambda}(A) \boldsymbol{x}^{\lambda}$. By the observation above, we have the following immediately.

Proposition 5.4. For any $\lambda$, it holds that

$$
G_{\lambda}(A)=\left(-A^{2}-A^{-2}\right)^{k} F_{\lambda}(A),
$$

where $k=\sum_{i \geq 1} \lambda_{i}-1$. In particular, $F_{0}(A)$ is divisible by $-A^{2}-A^{-2}$.

\section{ACKNOWLEDGMENTS}

The authors would like to thank Professors Yasutaka Nakanishi and Takuji Nakamura for many helpful suggestions and comments. The authors are also grateful to Yuki Okada for his help in this study.

\section{REFERENCES}

[1] D. M. Afanasiev and V. O. Manturov, On virtual crossing number estimates for virtual links, J. Knot Theory Ramifications 18 (2009), no. 6, 757-772. MR.2542694 (2010j:57011)

[2] H. A. Dye and L. H. Kauffman, Virtual crossing number and the arrow polynomial, J. Knot Theory Ramifications 18 (2009), no. 10, 1335-1357. MR2583800 (2010m:57011)

[3] R. Fenn, L. H. Kauffman, and V. O. Manturov, Virtual knot theory - unsolved problems, Fund. Math. 188 (2005), 293-323. MR2191949 (2006k:57011)

[4] J. Green, A Table of Virtual Knots, http://www.math.toronto.edu/drorbn/Students/GreenJ/

[5] A. Ishii, The pole diagram and the Miyazawa polynomial, International J. of Math. 19 (2008), 193-207. MR2384899 (2009m:57022)

[6] N. Kamada, An index of enhanced state of a virtual link diagram and Miyazawa polynomials, Hiroshima Math. J. 37 (2007), 409-429. MR2376727 (2008k:57025)

[7] L. H. Kauffman, State models and the Jones polynomial, Topology 26 (1987), no. 3, 395-407. MR899057 (88f:57006)

[8] L. H. Kauffman, Virtual knot theory, European J. Combin. 20 (1999), no. 7, 663-690. MR1721925 (2000i:57011)

[9] L. H. Kauffman, Detecting virtual knots, Atti Sem. Mat. Fis. Univ. Modena 49 (2001), suppl., 241-282. MR1881100 (2002k:57010) 
[10] T. Kishino, On classification of virtual links whose crossing numbers are equal to or less than 6 (in Japanese), Master Thesis, Osaka City University (2000).

[11] V. O. Manturov, Teoriya uzlov, in Regular and Chaotic Dynamics, Moscow-Izhevsk, 2005.

[12] V. O. Manturov, Minimal diagrams of classical and virtual links, arXiv:math/0501393

[13] Y. Miyazawa, A multi-variable polynomial invariant for virtual knots and links, J. Knot Theory Ramifications 17 (2008), no. 11, 1311-1326. MR2469206 (2009h:57020)

[14] Y. Miyazawa, A virtual link polynomial and the virtual crossing number, J. Knot Theory Ramifications 18 (2009), no. 5, 605-623. MR2527679 (2010j:57018)

[15] K. Murasugi, Jones polynomials and classical conjectures in knot theory, Topology 26 (1987), no. 2, 187-194. MR895570 (88m:57010)

[16] M. Thistlethwaite, A spanning tree expansion of the Jones polynomial, Topology 26 (1987), no. 3, 297-309. MR899051 (88h:57007)

[17] V.G. Turaev, A simple proof of the Murasugi and Kauffman theorems on alternating links, Enseign. Math. (2) 33 (1987), no. 3-4, 203-225. MR.925987 (89e:57002)

Department of Mathematics, Kobe University, Rokkodai-Cho 1-1, Nada-Ku, Kobe 657 0013, JAPAN

E-mail address: shin@math.kobe-u.ac.jp

Department of Mathematics, Kobe University, Rokkodai-Cho 1-1, Nada-ku, Kobe 657 0013, JAPAN 